Autoimmune (lgG4-related) Pancreatitis and Cholangitis 

Michael J. Levy • Suresh T. Chari Editors

\section{Autoimmune (IgG4-related) Pancreatitis and Cholangitis}

算 Springer 
Editors

Michael J. Levy

Division of Gastroenterology and Hepatology

Mayo Clinic

Rochester, MN, USA

\author{
Suresh T. Chari \\ Division of Gastroenterology and \\ Hepatology \\ Internal Medicine \\ Mayo Clinic College of Medicine \\ Rochester, MN, USA
}

\author{
ISBN 978-1-4419-6429-8 \\ DOI 10.1007/978-1-4419-6430-4 \\ Springer New York Heidelberg Dordrecht London \\ ISBN 978-1-4419-6430-4 (eBook)
}

\section{(C) Springer Science+Business Media New York 2013}

This work is subject to copyright. All rights are reserved by the Publisher, whether the whole or part of the material is concerned, specifically the rights of translation, reprinting, reuse of illustrations, recitation, broadcasting, reproduction on microfilms or in any other physical way, and transmission or information storage and retrieval, electronic adaptation, computer software, or by similar or dissimilar methodology now known or hereafter developed. Exempted from this legal reservation are brief excerpts in connection with reviews or scholarly analysis or material supplied specifically for the purpose of being entered and executed on a computer system, for exclusive use by the purchaser of the work. Duplication of this publication or parts thereof is permitted only under the provisions of the Copyright Law of the Publisher's location, in its current version, and permission for use must always be obtained from Springer. Permissions for use may be obtained through RightsLink at the Copyright Clearance Center. Violations are liable to prosecution under the respective Copyright Law.

The use of general descriptive names, registered names, trademarks, service marks, etc. in this publication does not imply, even in the absence of a specific statement, that such names are exempt from the relevant protective laws and regulations and therefore free for general use.

While the advice and information in this book are believed to be true and accurate at the date of publication, neither the authors nor the editors nor the publisher can accept any legal responsibility for any errors or omissions that may be made. The publisher makes no warranty, express or implied, with respect to the material contained herein.

Printed on acid-free paper

Springer is part of Springer Science+Business Media (www.springer.com) 


\section{Preface}

It is not very often that a new disease entity is discovered. While autoimmune pancreatitis was christened in 1995 , it is only in the last few years that the terms type 2 autoimmune pancreatitis and IgG4-related disease have been coined to describe new disease entities. So it is with great pleasure that we present this book titled IgG4-related disease (IgG4-RD). Our goal is to provide a timely overview of this disease entity with particular emphasis on its most well-described manifestations, namely, autoimmune pancreatitis (AIP) and IgG4-related sclerosing cholangitis (IgG4-SC).

As gastroenterologists, this effort grew out of our interest in AIP and IgG4-SC. As worldwide experience has grown, AIP and IgG4-SC are now recognized as manifestations of a multiorgan disease process termed IgG4related disease (IgG4-RD). While there is much overlap in terms of the clinical, serological, histological, and imaging features among the various organs involved, there are also key differences. Clearer understandings of the overlapping and disparate features have substantially aided patient care and are reviewed in detail throughout this book.

Initial efforts around the world occurred in isolation producing often conflicting evaluation and management strategies, leading to confusion and uncertainty among physicians. Recent multinational collaborative efforts have enhanced our understanding of the pathogenesis as well as clinical, laboratory, and imaging findings associated with IgG4-RD. This combined effort has led to the adoption of a broadly accepted nomenclature and helped standardize the diagnostic and therapeutic approach. However, in this book we do elaborate on key differences that remain throughout the world, thereby allowing clinicians and researchers to tailor their practice and studies accordingly. This book offers a manageable understanding of key aspects of the disease while providing in-depth information that should well serve those seeking a deeper understanding of this unique disorder.

We would like to acknowledge the renowned team of experts that we feel fortunate to have worked with, and we appreciate their hard work and devotion to this project. We have found this journey of discovery to be rewarding and hope you will as well.

Rochester, MN, USA

Michael J. Levy

Suresh T. Chari 



\section{Contents}

1 Background and Perspective .................................................. 1

Daniel S. Longnecker

\section{Part I Autoimmune Pancreatitis}

2 Immune Pathogenesis

Edward Alabraba, Shameena Bharucha, Penny Watson, and Robert Sutton

3 Overview of Types 1 and 2

Tooru Shimosegawa

4 Histologic Features

Günter Klöppel and Thomas C. Smyrk

5 CT and MRI Features

Naoki Takahashi and Dushyant V. Sahani

6 EUS Features.

Michael J. Levy and William R. Brugge

7 ERCP Features

Terumi Kamisawa

8 Clinical Features

Timothy B. Gardner and Chris E. Forsmark

9 Approach to Diagnosis

Raghuwansh P. Sah and Suresh T. Chari

10 Surgical Implications

Clancy J. Clark, Carlos Fernandez-del Castillo, and Michael B. Farnell

11 Approach to Therapy

Phil A. Hart and Suresh T. Chari

\section{Part II IgG4-Related Sclerosing Cholangitis}

12 Secondary Sclerosing Cholangitis

Gideon M. Hirschfield 
13 Histologic Features.

Lizhi Zhang and Vikram Deshpande

14 CT and MRI Features

Ali D. Karaosmanoglu, Naoki Takahashi, and Dushyant V. Sahani

15 ERCP and EUS/IDUS Features

George Webster and Atsushi Irisawa

16 Clinical Spectrum and Management

Einar S. Björnsson and Keith D. Lindor

\section{Part III Other Organ Involvement}

17 Overview

Raghuwansh P. Sah and Suresh T. Chari

18 Tubulointerstitial Nephritis and Other Renal Involvement by IgG4-Related Disease.

Lynn D. Cornell and Naoki Takahashi

19 IgG4-Related Lung Disease.

Jay H. Ryu, Hiroshi Sekiguchi, and Eunhee S. Yi

Part IV World-Wide Experience with AIP and IgG4-Related Sclerosing Cholangitis

20 Italian Experience

Luca Frulloni and Giuseppe Zamboni

21 British Experience

Evangelos Kalaitzakis, Robert Sutton, and George Webster

22 Japanese Experience

Kazuichi Okazaki and Kazushige Uchida

23 Korean Experience

Myung-Hwan Kim and Sung-Hoon Moon

24 Summary and Look to the Future

William R. Brugge and Markus M. Lerch

Index 


\section{Contributors}

Edward Alabraba NIHR Pancreas Biomedical Research Unit, Royal Liverpool University Hospital, Liverpool, UK

Shameena Bharucha NIHR Pancreas Biomedical Research Unit, Royal Liverpool University Hospital, Liverpool, UK

Einar S. Björnsson Landspitali University Hospital, Reykjavik, Iceland Department of Internal Medicine, Division of Gastroenterology, The National University Hospital of Iceland, Reykjavik, Iceland

William R. Brugge Harvard Medical School, Massachusetts General Hospital, Boston, MA, USA

Suresh T. Chari Division of Gastroenterology and Hepatology, Internal Medicine, Mayo Clinic College of Medicine, Rochester, MN, USA

Clancy J. Clark Wake Forest Baptist Health, Medical Center Blvd., Winston-Salem, NC, USA

Lynn D. Cornell Laboratory Medicine and Pathology, Mayo Clinic, Rochester, MN, USA

Vikram Deshpande Department of Pathology, Massachusetts General Hospital, Boston, MA, USA

Michael B. Farnell Mayo Clinic, Rochester, MN, USA

Carlos Fernandez-del Castillo Department of Surgery, Massachusetts General Hospital, Boston, MA, USA

Chris E. Forsmark Division of Gastroenterology, Hepatology, and Nutrition, University of Florida, Gainesville, FL, USA

Luca Frulloni Department of Medicine, University of Verona, Verona, Italy

Timothy B. Gardner Gastroenterology and Hepatology, Dartmouth Medical School, Lebanon, NH, USA

Phil A. Hart Division of Gastroenterology and Hepatology, Internal Medicine, Mayo Clinic College of Medicine, Rochester, MN, USA 
Gideon M. Hirschfield Centre for Liver Research, NIHR Biomedical Research Unit, University of Birmingham, Institute of Biomedical Research, Birmingham, UK

Atsushi Irisawa Department of Gastroenterology, Fukushima Medical University Aizu Medical Center, Aizuwakamatsu, Japan

Evangelos Kalaitzakis Department of Gastroenterology, Skåne University Hospital, Lund, Sweden

Terumi Kamisawa Department of Internal Medicine, Tokyo Metropolitan Komagome Hospital, Honkomagome, Bunkyo-ku/Tokyo, Japan

Ali D. Karaosmanoglu Department of Radiology, Massachusetts General Hospital (ADK, DS), Boston, MA, USA

Myung-Hwan Kim Department of Internal Medicine, University of Ulsan College of Medicine, Asan Medical Center, Seoul, South Korea

Günter Klöppel Department of Pathology, University Hospital, University of Kiel, Munich, Bavaria, Germany

Markus M. Lerch Department of Medicine, University Hospital Greifswald, Greifswald, Germany

Michael J. Levy Division of Gastroenterology and Hepatology, Mayo Clinic, Rochester, MN, USA

Keith D. Lindor Gastroenterology and Hepatology, Mayo Clinic, Rochester, MN, USA

Daniel S. Longnecker Department of Pathology, Geisel School of Medicine at Dartmouth, Dartmouth-Hitchcock Medical Center, Lebanon, NH, USA

Sung-Hoon Moon Department of Internal Medicine, Hallym University Sacred Heart Hospital, Hallym University College of Medicine, Anyang-si, South Korea

Kazuichi Okazaki Department of Gastroenterology and Hepatology, Kansai Medical University, Hirakata, Osaka, Japan

Jay H. Ryu Division of Pulmonary and Critical Care Medicine, Mayo Clinic College of Medicine, Rochester, MN, USA

Raghuwansh P. Sah Internal Medicine, Mayo Clinic Rochester, Rochester, MN, USA

Dushyant V. Sahani Department of Radiology, Massachusetts General Hospital (ADK, DS), Boston, MA, USA

Hiroshi Sekiguchi Division of Pulmonary and Critical Care Medicine, Mayo Clinic Rochester, Rochester, MN, USA

Tooru Shimosegawa Department of Gastroenterology, Hohoku University Hospital, Sendai, Miyagi, Japan 
Thomas C. Smyrk Department of Pathology, Mayo Clinic, Rochester, MN, USA

Robert Sutton NIHR Pancreas Biomedical Research Unit, Royal Liverpool University Hospital, Liverpool, UK

Naoki Takahashi Department of Radiology, Mayo Clinic, Rochester, MN, USA

Kazushige Uchida Department of Gastroenterology and Hepatology, Kansai Medical University, Hirakata, Osaka, Japan

Penny Watson Department of Veterinary Medicine, Queen's Veterinary School Hospital, University of Cambridge, Cambridge, Cambs, UK

George Webster GI Services, University College London Hospitals, London, UK

Eunhee S. Yi Department of Laboratory Medicine and Pathology, Mayo Clinic, Rochester, MN, USA

Giuseppe Zamboni Servizio di Anatomia-Istologia Patologica, Università di Verona, Ospedale S.Cuore-Don Calabria, Negrar-Verona, Italy

Lizhi Zhang Department of Laboratory and Anatomic Pathology, Mayo Clinic, Rochester, MN, USA 